\title{
Investigation of Open Circuit Potential of Lithium-Ion Battery by The Taguchi Design
}

\author{
Uğur Moral1
}

0000-0001-7794-6943

Chemical Engineering Department, Eskişehir Osmangazi University, Eskişehir, 26480, Turkey

\section{Abstract}

Lithium-ion batteries are widely used in electric vehicles. A robust battery management system is a very important issue for lithium-ion batteries due to the safety of electric vehicles. In this study, simultaneous main influences of charging factors on open circuit potential were statistically determined by using the Taguchi design. The response table and variance analysis were performed to determine the significant charging factors on a lithiumion battery. The results showed that the battery potential was the most influential charging factor to the open circuit potential. Furthermore, the statistically significant correlation between each charging factor and open circuit potential was determined based on a $95 \%$ significance level. The interactions between charging factors were demonstrated by the non-parallel lines presented on the interaction plots. The findings can guide the battery management and control methods for lithium-ion batteries used in electric vehicles.

Keywords: Battery Management, Charging Protocol, Lithium-ion Battery, Statistical Analysis

\section{Research Article}

https://doi.org/10.30939/ijastech..868549

$\begin{array}{ll}\text { Received } & 26.01 .2021 \\ \text { Revised } & 27.04 .2021 \\ \text { Accepted } & 28.04 .2021\end{array}$

Accepted 28.04.202

* Corresponding author

Uğur Moralı

umorali@ogu.edu.tr

Address: Chemical Engineering Department, Eskişehir Osmangazi University, Eskişehir, Turkey

Tel:+903122028653

\section{Introduction}

Lithium-ion batteries are one of the most preferred energy storage materials with their high energy density for numerous applications, including electric vehicles, hybrid electric vehicles, mobile phones, notebooks, battery-powered vehicles [1-4]. Performance of the lithium-ion batteries in the vehicle systems determine the safety, durability, reliability, and efficiency [5]. Equivalent circuit models are used to estimate the performance of lithium-ion cells due to concerning battery dynamic parameters. To improve the reliability of model-based battery management systems, equivalent circuit models have been extensively investigated, as reported in [6-10]. There are generally two terms that define the state of lithium-ion batteries, namely state-ofcharge and state-of-health [11]. The state-of-charge can be defined as the quantified value of the usable energy of battery, while the remaining performance is indicated by the state-of-health [12]. In the literature, the studies have also been performed to determine the state-ofcharge and state-of-health [8, 13]. Battery management systems use the term state-of-charge to observe the state of the battery and avoid over-charging or over-discharging [14]. The state-of-charge as a substantial parameter for a battery management system can be estimated if the battery's open circuit potential is known. The open circuit potetial thermodynamically reflects the battery's equilibrium potential under zero-current loading. Therefore, the open circuit potential is crucial to design a robust battery management system in real world operating conditions.
Charging operation is also important for battery-powered vehicles [15]. The constant current constant potential charging procedure consists of two steps, namely constant current mode, and constant potential mode [16]. In the constant current step, a constant charging current is applied to the lithium-ion battery until its potential reaches a cut-off voltage. The charging current gradually decreases when the battery potential is reached the cut-off voltage. The battery potential is kept constant during the constant potential mode while the charging current is decreased to a cut-off current that still charges the battery [17]. There are various charging factors influencing the battery characteristics during their life cycle. Charging current is one of the most important controllable factor. High charge currents provide a short charging time, while low charge currents are usually suggested by the battery manufacturers considering safety restrictions [18]. Another controllable charging factor is the relaxation time in which the battery potential is allowed to relax to its equilibrium potential. The effects of these charging factors on battery dynamic parameters have been studied in the literature [18, 19]. Schmidt et al. have charged the lithium-ion battery with a pulse current of $1 \mathrm{C}$ to different battery potentials [20]. The charging current was applied during $10 \mathrm{~s}$. They did not observe selfdischarge between 10-60\% state-of-charge. The self-discharge was clearly observed after approximately $60 \%$ state-of-charge. They proposed a model to predict the full-cell potential and half-cell potential. However, they did not statistically determine the influence of charging factors on the open circuit potential. In a recent work, Shkrob et al. 
have investigated the relaxation dynamics of fast charging of lithiumion batteries [21]. They observed that the charging current had a stronger influence on the potential relaxation behavior than the stateof-charge. However, the influence of both charging current and stateof-charge was not present statistically. However, the simultaneous effects of charging factors on the open circuit potential of lithium-ion batteries have not been investigated. This is likely due to that the determination of the simultaneous effects of charging factors on the open circuit potential, which requires both electrochemical and statistical analyses, is very difficult to interpret. These studies in the open literature have been done in a classical way in terms of experimental design terminology. The Taguchi design provide statistical results enabling an easy interpretation of factor effects. Thus, influence of each factor can be determined statistically by using the Taguchi design. Furthermore, the significant factors can be determined based on the variance analysis. This enables a quantitative determination of the association between the factors and the response variable. Moreover, the interactions between factors can be determined by the interaction plots. These results obtainable by the Taguchi design make this method preferable.

This study aims to provide quantification of the simultaneous influences of charging factors on open circuit potential for a robust battery management system. The charging factors were the charging current at constant-current state, battery potential, and relaxation time. The relative significance of each charging factor was determined based on the signal-to-noise ratio in the response table. Furthermore, the statistically significant association between the charging factors and the open circuit potential was demonstrated by the variance analysis. In addition, the interactions between charging factors are determined to provide a detailed understanding of the influences of charging current and relaxation time under certain battery potentials.

\section{Experimental Methods}

Commercial 2032 lithium-ion coin cell was used to perform the charging protocols. The specifications of 2032 lithium-ion button cell are presented in Table 1. The charging protocol was implemented between the available potential ranges of the lithium-ion cell (LIC). Constant current constant potential (CCCV) charging protocol was implemented to charge the LIC. The CCCV charging procedure as a universal method is recommended by the battery manufacturers [22]. This charging protocol has been widely used to perform the charging experiments in the literature because of its high efficiency and sufficient protection [23]. Therefore, the $\mathrm{CCCV}$ charging procedure was preferred to perform the charging experiments. The LIC was charged up to a cut-off potential at a constant current. After the potential was reached the cut-off potential, the charging current at $\mathrm{CV}$ mode was gradually decreased to the cut-off current of $1 \mathrm{~mA}$. Then, the LIC was allowed to reach the thermodynamic equilibrium potential. During this relaxation period, the open circuit potential measurement was performed. All experiments were performed by using Gamry Reference 3000 Potentiostat and its front panel connected to a desktop computer. This device provides to perform electrochemical testing in energy storage materials such as batteries, fuel cells, supercapacitors and so on. The Gamry Framework software and Echem Analyst were used to conduct the charging runs and to collect the data, respectively. The 2032 battery holder was used to obtain reliable results from the charging experiments. This holder enabled a direct connection of the 2032-coin battery cell to the potentiostat. The experimental setup is shown in Figure 1 schematically.

Table 1. Characteristics of 2032 lithium-ion coin cell

\begin{tabular}{c|c}
\hline Specification & Value \\
\hline Charging cut-off potential & $4.20 \mathrm{~V}$ \\
\hline Discharging cut-off potential & $3.00 \mathrm{~V}$ \\
\hline Diameter & $20 \mathrm{~mm}$ \\
\hline Thickness & $3.2 \mathrm{~mm}$ \\
\hline
\end{tabular}

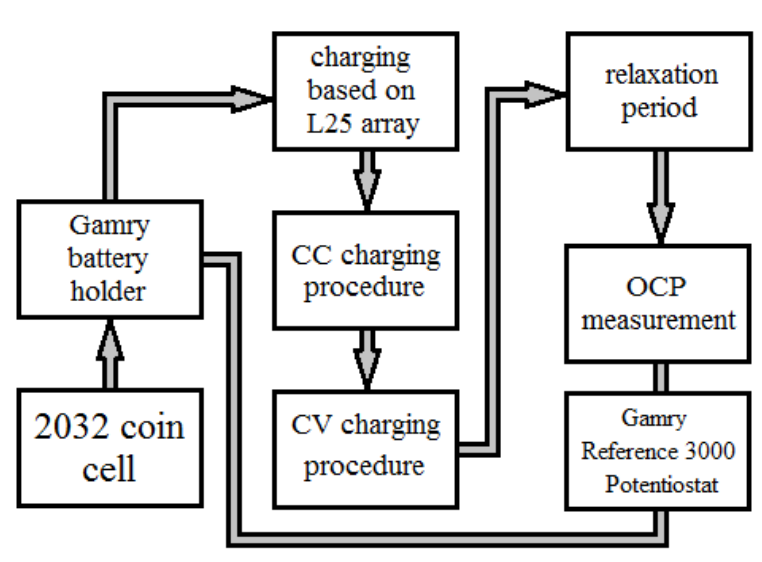

Fig. 1. A schematic representation of the experimental setup used to perform the charging runs

The charging conditions implemented were determined by using the Taguchi design, in which the charging current at the $\mathrm{CC}$ and $\mathrm{CV}$ modes were varying. In addition, different levels of relaxation time $\left(\mathrm{t}_{\mathrm{rlx}}\right)$ were used during the open circuit potential measurements. The charging protocol are presented as an orthogonal matrix in Table 2. The capacity of the 2032 battery cell of 31.75 $\mathrm{mAh}$ was experimentally determined by using the Coulomb counting method. Thus, the implemented charging current of $31.75 \mathrm{~mA}$ represents $1 \mathrm{C}$-rate due to the charging process is completed in 1 hour. The orthogonal array $\mathrm{L} 25\left(5^{3}\right)$ enabled to determine the main effects and interaction influences of control factors [24].

\section{Results and Discussion}

The LIC was charged up to the cut-off potential and followed by a constant potential charging mode. The open circuit potential measurements showed that the applied charging protocols influenced the final potential of LIC. The final potential was the battery potential at the end of relaxation period. The open circuit potential difference between the cut-off potential, which is intended to be the ultimate battery potential, and the final potential is presented in Figure 2. 
Table 2. Steps of charging protocol based on orthogonal array.

\begin{tabular}{|c|c|c|c|}
\hline Run & charging current, mA & potential, V & $\mathrm{t}_{\mathrm{rlx}}, \mathrm{h}$ \\
\hline 1 & 63.50 & 3.7 & 0.50 \\
\hline 2 & 63.50 & 3.8 & 0.75 \\
\hline 3 & 63.50 & 3.9 & 1.00 \\
\hline 4 & 63.50 & 4.0 & 1.25 \\
\hline 5 & 63.50 & 4.2 & 1.50 \\
\hline 6 & 31.75 & 3.7 & 0.75 \\
\hline 7 & 31.75 & 3.8 & 1.00 \\
\hline 8 & 31.75 & 3.9 & 1.25 \\
\hline 9 & 31.75 & 4.0 & 1.50 \\
\hline 10 & 31.75 & 4.2 & 0.50 \\
\hline 11 & 15.88 & 3.7 & 1.00 \\
\hline 12 & 15.88 & 3.8 & 1.25 \\
\hline 13 & 15.88 & 3.9 & 1.50 \\
\hline 14 & 15.88 & 4.0 & 0.50 \\
\hline 15 & 15.88 & 4.2 & 0.45 \\
\hline 16 & 3.97 & 3.7 & 1.25 \\
\hline 17 & 3.97 & 3.8 & 1.50 \\
\hline 18 & 3.97 & 3.9 & 0.50 \\
\hline 19 & 3.97 & 4.0 & 0.75 \\
\hline 20 & 3.97 & 4.2 & 1.00 \\
\hline 21 & 1.98 & 3.7 & 1.50 \\
\hline 22 & 1.98 & 3.8 & 0.50 \\
\hline 23 & 1.98 & 3.9 & 0.75 \\
\hline 24 & 1.98 & 4.0 & 1.00 \\
\hline 25 & 1.98 & 4.2 & 1.25 \\
\hline
\end{tabular}

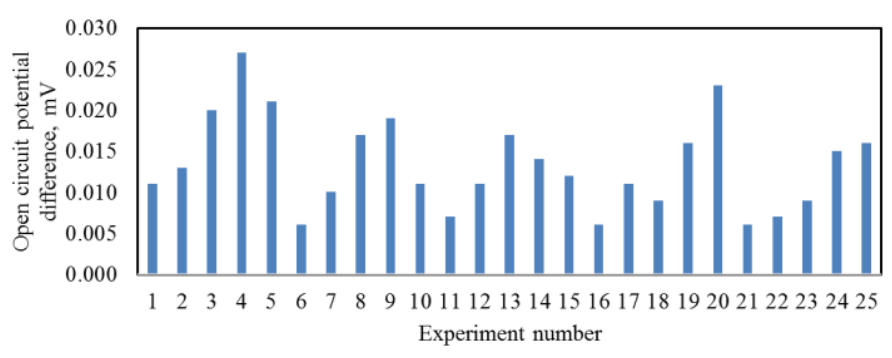

Fig. 2. Effect of charging factors on open circuit potential difference

The results presented in Figure 2 are difficult to interpret by using conventional experimental methodologies because the Taguchi design does not follow one factor at a time approach. Therefore, the effect of each charging factor on the open circuit potential was determined and interpreted by using response table and variance analysis (ANOVA). The response table for signal-to-noise ratios $(\mathrm{S} / \mathrm{N})$ is presented in Table 3 . After the implemented charging protocol, the decrease in the potential of LIC is not intended during the rest period. Thus, the $\mathrm{S} / \mathrm{N}$ value of each charging factor was calculated by using smaller-is-better quality assurance. This quality assurance indicated the influence of each charging factor to minimize the decrease in the potential during the rest period. In other words, the effect of the charging factors was quantified by the response table. The $\mathrm{S} / \mathrm{N}$ ratios and delta values are presented in Table 3. Delta can be calculated by the difference between the highest $\mathrm{S} / \mathrm{N}$ ratio and the smallest $\mathrm{S} / \mathrm{N}$ ratio. The highest $\mathrm{S} / \mathrm{N}$ ratio and the smallest $\mathrm{S} / \mathrm{N}$ ratio for the potential were 43.12 and 35.07, respectively. Thus, the delta value of the potential was 8.06. Concerning the relaxation time, the $\mathrm{S} / \mathrm{N}$ ratio of level 1 was 39.89 . The lowest $\mathrm{S} / \mathrm{N}$ ratio of the relaxation time was obtained as 37.26. The delta value of 2.63 was obtained by the difference between these $\mathrm{S} / \mathrm{N}$ ratios of the relaxation time. The delta values of the charging current was 5.01. The potential showed the highest delta value. The highest delta value of the potential indicated that the effect of the potential on the open circuit potential difference was higher than the other charging factors. The delta value of the charging current (5.01) was higher than the relaxation time (2.63). This showed that the influence of the charging current was approximately two orders of magnitude higher than that of the relaxation time. In other words, the relaxation time was the least effective charge factor on the open circuit potential difference compared to other charge factors.

Table 3. Response table for signal-to-noise ratios - smaller is better.

\begin{tabular}{c|c|c|c}
\hline Level & Charging current & Potential & Relaxation time \\
\hline 1 & 40.17 & 43.12 & 39.89 \\
\hline 2 & 38.64 & 39.83 & 39.48 \\
\hline 3 & 38.63 & 37.32 & 37.26 \\
\hline 4 & 38.69 & 35.05 & 37.26 \\
\hline 5 & 35.16 & 35.97 & 37.40 \\
\hline Delta & 5.01 & 8.06 & 2.63 \\
\hline Rank & 2 & 1 & 3 \\
\hline
\end{tabular}

The ANOVA was used to determine if the association between the charging factors and open circuit potential difference was statistically significant based on the significance level of 95\%. Probability $(\mathrm{P})$ value of each charging factor is presented in Table 4. The lowest P-value of 0.000 was obtained for the potential. On the other hand, the $\mathrm{P}$-values of the charging current and the relaxation time were 0.004 and 0.022 , respectively. The P-value of each charging factor less than 0.05 indicated that all of the charging factors should be carefully controlled during the charging protocol [25]. In addition, these P-values demonstrated that there was a statistically significant association between the charging factors and the open circuit potential difference. The minimum P-value of 0.000 showed that the charging current was the most significant factor on the open circuit potential difference, as also shown by the response table. Furthermore, the highest F-value of 15.70 was obtained for the potential. The F-value of 6.74 obtained for the charging current was between the F-values of the potential and the relaxation time. This showed that the effect of the charging current was lower than that of the potential and higher than that of the relaxation time. The results from the response table and the ANOVA demonstrated that the potential was the most important factor. However, it is important to emphasize that the influences of the 
charging current and the relaxation time should be monitored during the charging process of the lithium-ion battery.

Table 4. Variance analysis for open circuit potential.

\begin{tabular}{c|c|c|c|c|c}
\hline Source & Seq SS & Adj SS & Adj MS & $\mathrm{F}$ & $\mathrm{P}$ \\
\hline $\begin{array}{c}\text { charging } \\
\text { current }\end{array}$ & 0.0001754 & 0.0001754 & 0.0000438 & 6.74 & 0.004 \\
\hline potential & 0.0004086 & 0.0004086 & 0.0001021 & 15.70 & 0.000 \\
\hline $\mathrm{t}_{\mathrm{rlx}}$ & 0.0001118 & 0.0001118 & 0.0000279 & 4.29 & 0.022 \\
\hline Error & 0.0000781 & 0.0000781 & 0.0000065 & & \\
\hline Total & 0.0007738 & & & & \\
\hline
\end{tabular}
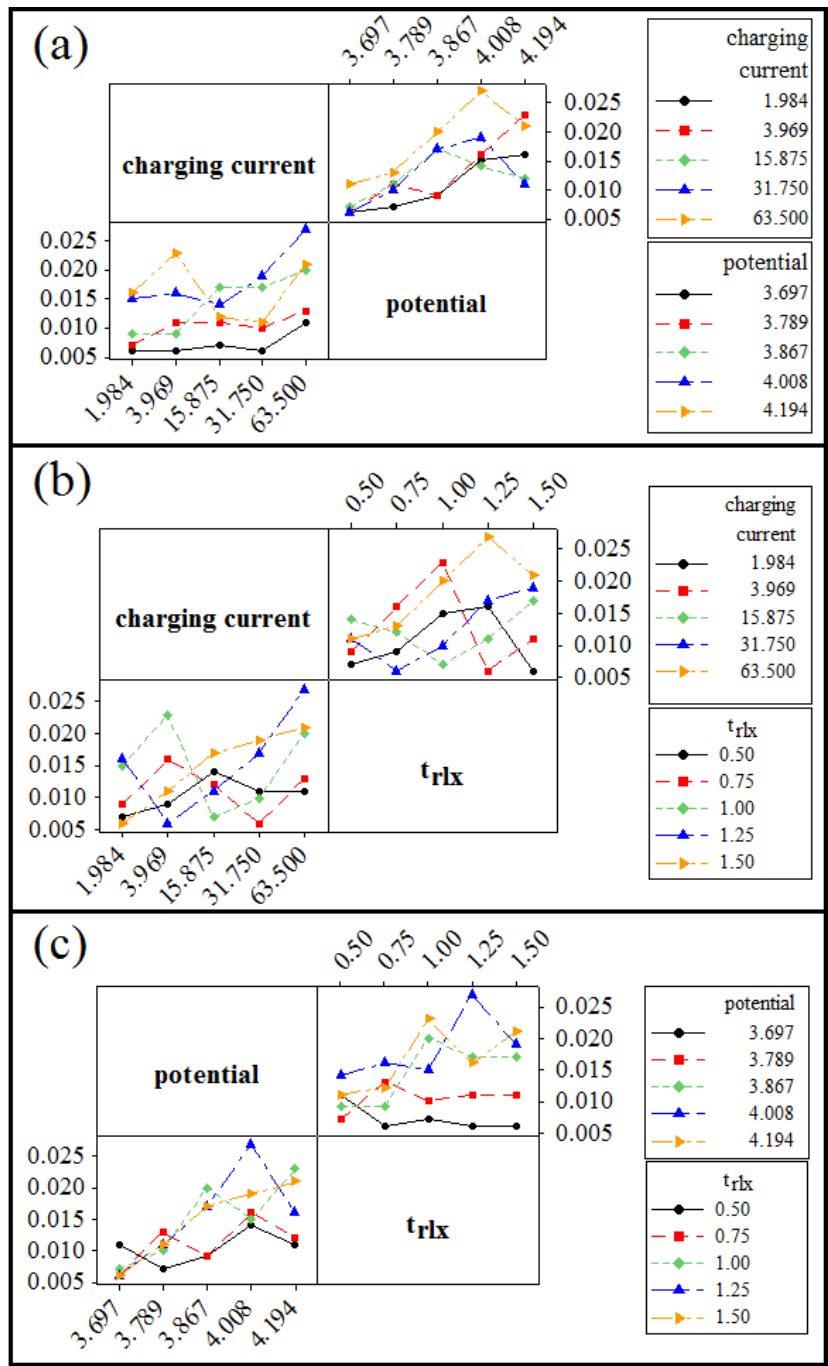

Fig. 3. Interaction plots of charging factors

Even though it is important to determine the main effect of each charging factor on open circuit potential difference, a robust battery management system needs more quantified data such as interactions between the control factors. In this work, the interactions between the charging factors was determined by using interaction plots. The interaction between charging factors shows how the relationship between a charging factor and open circuit potential dif- ference depends on the level of other charging factors. The interaction between each charging factors is plotted and presented in Figure 3. Non-parallel lines on the plots demonstrated that there were interactions between the charging factors and open circuit potential difference. It was depicted in Figure $3 \mathrm{a}$ that the correlation between the charging current and open circuit potential difference depended on the level of the potential. Similar intersections between the lines depicted in Figure $3 \mathrm{~b}$ and $3 \mathrm{c}$ indicated the interaction effects between the charging factors.

Summing up, the main effects and the interaction effects showed that the charging factors investigated in this work should be closely monitored during the charging process to achieve the desired battery potential.

\section{Conclusions}

The simultaneous influences of the charging factors on the open circuit potential difference have been determined by the Taguchi design and statistical analysis. The battery potential was the most influential charging factor due to its highest delta value of 8.06. The influence of the charging current was approximately two orders of magnitude higher than that of the relaxation time. The Pvalues lower than 0.05 obtained for each charging factor demonstrated that there was a statistically significant association between the charging factors and open circuit potential difference. The lowest $\mathrm{P}$-value of 0.000 obtained for the battery potential indicated the significance of the potential as a factor to obtain the desired battery potential during the proposed charging process. The higher F-value of the charging current than the relaxation time indicated stronger influence of the charging current. Furthermore, each significant charging factor influenced the effect of other charging factors on the open circuit potential difference. As a conclusion, the main influences of each charging factor and the determined interactions between these factors should be taken into account to achieve a robust battery management system.

\section{Acknowledgment}

This study is supported by Scientific Research Foundation (Eskisehir Osmangazi University) Project Number: 2017-1911.

\section{Conflict of Interest Statement}

The author declares that there is no conflict of interest.

\section{References}

[1] Barai A, Widanage WD, Marco J, McGordon A, Jennings P. A study of the open circuit voltage characterization technique and hysteresis assessment of lithium-ion cells. Journal of Power Sources. 2015;295:99-107.

[2] Zhang SS, Xu K, Jow TR. Study of the charging process of a $\mathrm{LiCoO}_{2}-$ based Li-ion battery. Journal of Power Sources. 2006;160(2):134954.

[3] Dermenci KB. Stability of Solid-State Sintered $\mathrm{Li}_{1.5} \mathrm{Al}_{0.5} \mathrm{Ge}_{1.5}\left(\mathrm{PO}_{4}\right)_{3}$ Solid Electrolytes in Various Mediums for All Solid-State Li-ion 
Batteries. International Journal of Automotive Science and Technology. 2020;4(4):295-9.

[4] Özgüç MK, Aras K. Selective Shut-off Strategy in Distributed Battery System. International Journal of Automotive Science and Technology. 2021;5(1):27-33.

[5] Xing Y, He W, Pecht M, Tsui KL. State of charge estimation of lithium-ion batteries using the open-circuit voltage at various ambient temperatures. Applied Energy. 2014;113:106-15.

[6] Erol S. Process Model Development of Lithium-ion Batteries-An Electrochemical Impedance Spectroscopy Simulation. Sakarya Üniversitesi Fen Bilimleri Enstitüsü Dergisi. 2020;24(6):1191-7.

[7] Erol S, Orazem ME. The influence of anomalous diffusion on the impedance response of $\mathrm{LiCoO}_{2} \mid \mathrm{C}$ batteries. Journal of Power Sources. 2015;293:57-64.

[8] Wang L, Lu D, Liu Q, Liu L, Zhao X. State of charge estimation for LiFePO4 battery via dual extended kalman filter and charging voltage curve. Electrochimica Acta. 2019;296:1009-17.

[9] Morali U, Erol S. The comparison of electrochemical impedance behaviors of lithium-ion and nickel-metal hydride batteries at different state-of-charge conditions. Journal of the Engineering and Architecture Faculty of Eskişehir Osmangazi University. 2020;28(1):1-8.

[10]Ekici YE, Nusret T. Charge and discharge characteristics of different types of batteries on a hybrid electric vehicle model and selection of suitable battery type for electric vehicles. International Journal of Automotive Science and Technology. 2018;3(4):62-70.

[11]Morali U, Erol S. Electrochemical impedance analysis of 18650 lithium-ion and 6HR61 nickel-metal hydride rechargeable batteries. Journal of the Faculty of Engineering and Architecture of Gazi University. 2020;35(1):297-310.

[12]Zheng F, Xing Y, Jiang J, Sun B, Kim J, Pecht M. Influence of different open circuit voltage tests on state of charge online estimation for lithium-ion batteries. Applied Energy. 2016;183:513-25.

[13]Cui Y, Zuo P, Du C, Gao Y, Yang J, Cheng X, et al. State of health diagnosis model for lithium ion batteries based on real-time impedance and open circuit voltage parameters identification method. Energy. 2018;144:647-56.

[14]Pei L, Wang T, Lu R, Zhu C. Development of a voltage relaxation model for rapid open-circuit voltage prediction in lithium-ion batteries. Journal of Power Sources. 2014;253:412-8.

[15]Solmaz H, Kocakulak T. Determination of Lithium Ion Battery Characteristics for Hybrid Vehicle Models. International Journal of Automotive Science and Technology. 2020;4(4):264-71.

[16]Eddahech A, Briat O, Vinassa J-M. Determination of lithium-ion battery state-of-health based on constant-voltage charge phase. Journal of Power Sources. 2014;258:218-27.

[17]Li Y, Li K, Xie Y, Liu J, Fu C, Liu B. Optimized charging of lithiumion battery for electric vehicles: Adaptive multistage constant current-constant voltage charging strategy. Renewable Energy. 2020;146:2688-99.

[18]Morali U. Synergistic influence of charge conditions on electrochemical impedance response of $\mathrm{LiNiMnCoO}_{2} \mid \mathrm{C}$ coin cells-Complementary statistical analysis. Journal of Energy Storage. 2020;32:101809.

[19]Morali U. Influence of charge conditions on battery dynamics of a commercial lithium-ion cell. Hacettepe Journal of Biology and Chemistry. 2020;48(3).
[20]Schmidt JP, Weber A, Ivers-Tiffée E. A novel and fast method of characterizing the self-discharge behavior of lithium-ion cells using a pulse-measurement technique. Journal of Power Sources. 2015;274:1231-8.

[21]Shkrob IA, Rodrigues M-TF, Abraham DP. Fast charging of Li-ion cells: Part III. Relaxation dynamics and trap-controlled lithium ion transport. Journal of The Electrochemical Society. 2019;166(16):A4168.

[22]Wang Z, Zeng S, Guo J, Qin T. State of health estimation of lithiumion batteries based on the constant voltage charging curve. Energy. 2019;167:661-9.

[23]Liu H, Naqvi IH, Li F, Liu C, Shafiei N, Li Y, et al. An analytical model for the CC-CV charge of $\mathrm{Li}$-ion batteries with application to degradation analysis. Journal of Energy Storage. 2020;29.

[24]Morali U, Demiral H, Sensoz S. Synthesis of carbon molecular sieve for carbon dioxide adsorption: Chemical vapor deposition combined with Taguchi design of experiment method. Powder Technology. 2019;355:716-26.

[25] Samet U. Multi-Objective Optimization of Biodiesel and Diethyl Ether Doped Diesel Engine by Taguchi Method. International Journal of Automotive Science and Technology. 2020;4(3):171-9. 